\title{
Predictors of the "Crosstalk" Phenomenon During Cryoballoon Ablation in Patients with Atrial Fibrillation Continue Freezing if Ablation Meets the Criteria
}

\author{
Tatsuhiko Hirao, ${ }^{1,2}$ MD, Yasuteru Yamauchi, ${ }^{1}$ MD, Rena Nakamura, ${ }^{1}$ MD, Takatoshi Shigeta, ${ }^{1}$ MD, \\ Hiroshi Yoshida, ${ }^{1}$ MD, Shinichi Tachibana, ${ }^{1}$ MD, Atsuhito Oda, ${ }^{1}$ MD, Aki Ito, ${ }^{1}$ MD, \\ Mitsutoshi Asano, ${ }^{1}$ MD, Hidetoshi Suzuki, ${ }^{1}$ MD, Tsukasa Shimura, ${ }^{1}$ MD, Manabu Kurabayashi, ${ }^{1}$ MD, \\ Masahiko Goya, ${ }^{2} \mathrm{MD}$, Kaoru Okishige, ${ }^{1,3} \mathrm{MD}$ and Tetsuo Sasano, ${ }^{2} \mathrm{MD}$
}

\begin{abstract}
Summary
Second-generation cryoballoon (CB) ablation is effective in achieving pulmonary vein (PV) isolation (PVI) in atrial fibrillation (AF) patients. The "crosstalk" (CST) phenomenon has been reported to reduce unnecessary applications during CB ablation. Nevertheless, it is unclear under what conditions the CST phenomenon occurs.

To seek the predictors of the CST phenomenon during CB-guided PVI, CST phenomenon in achieving ipsilateral superior PVI during inferior PV ablation was analyzed in AF patients who underwent de novo ablation using CB. CB occlusion status and nadir balloon temperature (NT) were compared in these patients, and all ablated superior PVs were categorized into three groups according to the necessity of the touch up ablation and effectiveness of the phenomenon.

Of 1082 superior PVs, 16, 40, and 1026 were classified into the CST success, CST failure, and control groups (unnecessary CST), respectively. The proportion of superior PVs ablated with complete occlusion using the CB was significantly higher in the CST success group than in the other two groups. The proportion of superior PVs ablated with $\mathrm{NT} \leq-46^{\circ} \mathrm{C}$ was higher in the CST success group than in the CST failure group. The CST phenomenon was always observed if CB ablation of the superior PVs was performed with both complete occlusion and $\mathrm{NT} \leq-46^{\circ} \mathrm{C}$ and was almost always ineffective if it did not meet these two criteria (sensitivity, $100 \%$; specificity, $93 \%$ ).

Successful CST ablation was highly predicted if complete PV occlusion and $\mathrm{NT} \leq-46^{\circ} \mathrm{C}$ during CB ablation of the superior PVs were achieved.
\end{abstract}

(Int Heart J 2021; 62: 320-328)

Key words: Catheter ablation, Electrical connection, Pulmonary vein isolation

$\mathrm{W}$ ith respect to efficacy, cryoballoon (CB)guided pulmonary vein $(\mathrm{PV})$ isolation of paroxysmal atrial fibrillation (AF) can provide a comparable ${ }^{1-4)}$ or better outcome than contact force-guided radiofrequency catheter ablation. ${ }^{5)}$ Thus, CB ablation is clearly one of the most effective treatment modalities for $\mathrm{AF}^{6,7)}$ However, $\mathrm{CB}$ application indicates the risk of severe PV stenosis/occlusions ${ }^{8.9}$ and may damage extracardiac structures such as the esophagus, ${ }^{10}$ phrenic nerve, and bronchus. ${ }^{11,12)}$

Chun, et al. reported the "crosstalk" (CST) phenomenon in which $\mathrm{CB}$ application of the left inferior PV (LIPV) was able to ablate the gap sites along the inferior aspect of the left superior PV (LSPV) when applications failed to isolate the LSPV ${ }^{13)}$ Where this CST phenomenon can be beneficial in terms of reducing unnecessary freezing of the atrial tissue, although this report was for a first- generation $\mathrm{CB}$, it is a phenomenon that we equally encounter when using a second-generation CB. Nevertheless, it is unclear which situations will necessitate the adoption of the CST phenomenon.

The present study aimed to investigate the relationship between the NT and occlusion status of the CB and the efficacy of CST ablation. Additionally, we sought to determine the predictors of the CST phenomenon under which conditions occur.

\section{Methods}

Study population: Patients with drug-refractory AF who underwent de novo $\mathrm{CB}$ ablation using second-generation CB from July 2017 to February 2020 were included in this retrospective, single-center, non-randomized study. While all patients provided informed consent prior to the

From the ${ }^{1}$ Heart Center, Yokohama-City Bay Red Cross Hospital, Yokohama, Japan, ${ }^{2}$ Department of Cardiovascular Medicine, Tokyo Medical and Dental University, Tokyo, Japan and ${ }^{3}$ Yokohama Minato Heart Clinic, Yokohama, Japan.

Address for correspondence: Yasuteru Yamauchi, MD, Heart Center, Yokohama-City Bay Red Cross Hospital, 3-12-1 Shinyamashita, Naka-ku, Yokohama, Kanagawa 231-8682, Japan. E-mail: yasuteru1020@gmail.com

Received for publication October 16, 2020. Revised and accepted December 1, 2020.

Released in advance online on J-STAGE March 17, 2021.

doi: 10.1536/ihj.20-690

All rights reserved by the International Heart Journal Association. 
ablation procedure, to establish the left atrial (LA) and PV anatomy, all patients underwent a computed tomography scan prior to ablation. We excluded those with left common PVs, huge PVs in which the CB was applied separately to the upper and lower parts of the PV, LA diameter $>55 \mathrm{~mm}$, intracardiac thrombi, uncontrolled thyroid dysfunction, contraindications for anticoagulation, and uncontrollable heart failure.

This study was conducted in accordance with the principles outlined in the Declaration of Helsinki. The study protocol was approved by the local institutional committee on human research at the Japan Redcross Yokohama-City Bay Hospital (approval number, 2017-81). Pre-ablation preparation protocol: Prior to the procedure, all antiarrhythmic drugs were discontinued for at least five half-lives. Direct oral anticoagulants were changed to dabigatran from the day before the ablation. Patients on warfarin continued taking it during the periprocedural period. An international normalized ratio level between 2.0 and 3.0 was considered acceptable. All patients continued taking anticoagulants throughout the periprocedural period. While surface electrocardiograms and bipolar intracardiac electrograms filtered from 30 to 500 $\mathrm{Hz}$ were monitored, using prone-positional enhanced computed tomography or intracardiac/transesophageal echocardiography, we confirmed that there were no intracardiac thrombi before ablation.

Ablation was performed under general anesthesia with dexmedetomidine or propofol. The ablation procedure has been previously described in detail. ${ }^{15)}$ Briefly, a standard transvenous approach was employed for transseptal puncture and subsequent PV isolation. A 7-Fr 20-pole three-site mapping catheter (BeeAT; Japan Lifeline, Tokyo, Japan) was located in the coronary sinus for anatomic guidance. With a radiofrequency needle (Baylis Medical, Montreal, QC, Canada), a single transseptal puncture of the fossa ovalis was performed using the Brockenbrough technique. The puncture was guided by intracardiac echocardiography (ACUSON AcuNav; Biosense Webster, Diamond Bar, CA, USA) and biplane fluoroscopy (right and left anterior oblique projections). Thereafter, heparin was administered intravenously to achieve an activated clotting time between 300 and 400 seconds during the ablation procedure.

Cryoballoon ablation protocol: After gaining LA access, we injected contrast into the LA to define the position of each PV ostium. Through a steerable 15-Fr sheath (FlexCath Advance; Medtronic, Minneapolis, MN, USA), an inner lumen mapping catheter (Achieve; Medtronic, Minneapolis, MN, USA) was advanced into each PV ostium. Subsequently, a 28-mm second-generation CB (Arctic Front Advance; Medtronic, Minneapolis, MN, USA) was advanced over the inner lumen mapping catheter, inflated, and positioned sequentially in the PV ostium of each vein. An optimal vessel occlusion was considered to be achieved upon selective contrast injection from the tip of the $\mathrm{CB}$ showing total contrast retention with no backflow into the LA. Once a complete occlusion was documented, we withdrew the $\mathrm{CB}$ slightly and allowed leakage around the PV-balloon interface to better define the PV ostium and ensured a proximal ablation position. We reapplied only the minimal amount of pressure needed to regain the occlusion before the ablation, and cryothermal energy delivery was commenced. Ten to fifteen seconds after the freezing started, we injected contrast from the tip of the $\mathrm{CB}$ again to confirm if the PV occlusion status changed due to increase of the internal pressure of the CB. Standard cryoenergy applications lasted for 180 to $240 \mathrm{sec}-$ onds. If PV potentials were visible during freezing energy delivery, the time to PV isolation was recorded when the potentials completely disappeared or became dissociated from the LA activity.

When the superior PV could not be isolated using a $\mathrm{CB}$, we expected the CST phenomenon and advanced the $\mathrm{CB}$ into the ipsilateral inferior PV. At this time, we inserted one more sheeth to LA through the same transseptal puncture site and positioned the decapolar ring catheter into the superior PV in order to monitor the potential of superior PV during freezing of the inferior PVs. When the time to PV isolation was clearly measured, we used it as a guide in deciding the freezing duration; we usually added more than 120 seconds to it as long as the total freezing duration did not exceed 240 seconds. If the superior PV was isolated during $\mathrm{CB}$ ablation of the ipsilateral inferior PV, we regarded this as successful CST phenomenon. When PV isolation was not achieved with $\mathrm{CB}$ application alone, we performed touch-up ablation of the PV using an open-irrigated radiofrequency catheter (FlexAbility and TactiCath, Abbott, Chicago, IL, USA, or ThermoCool, Biosense Webster, Irvine, CA, USA). CB ablation was applied in the following order: LSPV, LIPV, right inferior PV, and right superior PV (RSPV).

During cryoablation of right-sided PVs, continuous phrenic nerve stimulation with a decapolar catheter positioned in the superior vena cava was performed. The integrity of the phrenic nerve was monitored by intermittent fluoroscopy, tactile feedback, and right-sided compound motor action potential (CMAP) monitoring. When the CMAP amplitude decreased by more than $30 \%$ from the control value, $\mathrm{CB}$ application was prematurely terminated to avoid any injury to the phrenic nerve.

During CB ablation, the luminal esophageal temperature was also continuously monitored using an esophageal temperature probe (SensiTherm, Abbott, Chicago, IL, USA, or Esophaster; Japan Lifeline, Tokyo, Japan). CB application was stopped when the esophageal temperature dropped below $15^{\circ} \mathrm{C}$.

Statistical analysis: Continuous variables are presented as mean \pm standard deviation or as median and interquartile range and were compared using the $t$-test or Wilcoxon rank-sum test based on the distribution. Categorical variables are expressed as number and percentage and were compared using the $\chi^{2}$ test. A $P<0.05$ was considered to indicate statistical significance. Multiple comparisons of continuous variables were performed using the Wilcoxon/ Kruskal-Wallis test, whereas multiple comparisons of numerous variables were conducted with the $\chi^{2}$ test. If the test indicated a significant difference, we performed posthoc analysis with adjustment using the Bonferroni method. 
Table I. Baseline Clinical Characteristics

\begin{tabular}{lc}
\hline Variable & \\
\hline$n$ & 541 \\
Age, years & $67(59-73)$ \\
Male, $n(\%)$ & $384(71 \%)$ \\
Paroxysmal AF, $n(\%)$ & $351(65 \%)$ \\
Body mass index, $\mathrm{kg} / \mathrm{m}^{2}$ & $24.0(21.4-26.9)$ \\
LA diameter, mm & $41(36-46)$ \\
LV ejection fraction, $\%$ & $65(59-71)$ \\
CHADS & $1.17(0-2)$ \\
CHA $_{2} \mathrm{DS}_{2}$-VASc & $2.36(2-3)$ \\
\hline
\end{tabular}

$\mathrm{AF}$ indicates atrial fibrillation; LA, left atrium; and LV, left ventricle.

\section{Results}

Patient characteristics: A total of 541 patients with AF (average age, $67.4 \pm 10.8$ years; $71 \%$, males) who underwent de novo catheter ablation using the secondgeneration CB were included in this study. The patients' baseline characteristics are summarized in Table I.

Classification of the PVs based on the outcome of CB ablation and CST phenomenon: Out of 1082 PVs, 56 superior PVs (31 LSPVs and 25 RSPVs) were not isolated by $\mathrm{CB}$ ablation of the superior $\mathrm{PV}$, and $\mathrm{CB}$ ablation of the inferior PV was attempted. Among these 56 superior PVs, the CST phenomenon was observed in $16 \mathrm{PVs}$ (CST success group) but ineffective in 40 PVs that required touch-up radiofrequency ablation to achieve PV isolation (CST failure group). The third group involved superior PVs that were isolated only with ablation targeting the superior PV ("unnecessary CST" group = control group). Thus, we categorized the ablated superior PVs into three groups according to the effects of $\mathrm{CB}$ ablation and CST phenomenon, as shown in Figure 1.

Comparisons of $\mathrm{CB}$ procedures among the CST success, CST failure, and control group: Two factors of $\mathrm{CB}$ applications to the superior PVs - namely, NT and percentage of complete occlusions - were compared among the three groups (Figure 2). Differences in the NT among the three groups are illustrated in Figure 2A; the NT was significantly lower in the CST success and control groups than in the CST failure group $(P<0.001)$. Of note, complete occlusion of the superior PVs with the CB was significantly more highly achieved in the CST success group than in the CST failure and control groups (Figure 2B).

We analyzed the NT distribution during incomplete and complete occlusion applications, as shown by the graph in Figure 3. The NT in the CST success group was always observed in the temperature range of $\leq-46^{\circ} \mathrm{C}$ and most of them met the condition of complete occlusion. In contrast, the NT in the CST failure group was widely scattered throughout all temperatures, and many of them were under the condition of incomplete occlusion. Some superior PVs could not be isolated if the NT was above $-45^{\circ} \mathrm{C}$, even if a complete $\mathrm{CB}$ occlusion was achieved. As suggested by this graph, the CST phenomenon was always observed if $\mathrm{CB}$ ablation of the superior PVs met two factors of both complete $\mathrm{CB}$ occlusion and NT $\leq$ $-46^{\circ} \mathrm{C}$. Conversely, the CST phenomenon was almost al-

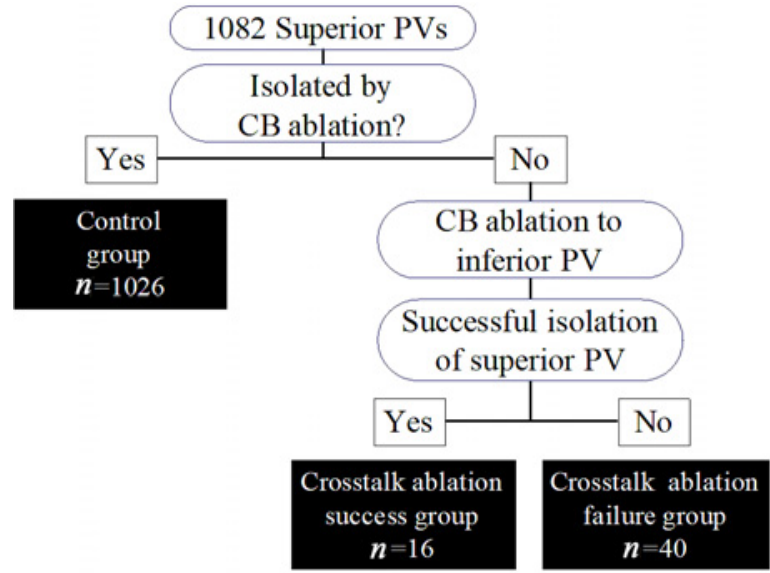

Figure 1. Patient distribution flowchart. The nadir balloon temperature and balloon occlusion status were analyzed and compared among the three groups. CB indicates cryoballoon; and PV, pulmonary vein.

ways ineffective if it did not meet both of these two criteria (sensitivity, 100\%; specificity, $93.8 \%$ ). When only the factor of complete PV occlusion was used as the criterion, the sensitivity and specificity were $93.8 \%$ and $87.5 \%$, respectively, which were worse than those of the above mentioned criteria.

Characteristics of the CB procedure in the CST success group: The characteristics of parameters during $\mathrm{CB}$ applications to the superior and inferior PVs in 16 cases in the CST success group are summarized in Table II. Complete occlusion of the superior PVs with the CB was achieved in 15/16 cases (93.8\%) and the minimum NT for $\mathrm{CB}$ application to the superior PVs ranged from $-46^{\circ} \mathrm{C}$ to $-60^{\circ} \mathrm{C}$. In all 16 cases except case nine, the first $\mathrm{CB}$ application to the inferior PVs succeeded in isolating the superior PVs, and no further applications were added. In case nine, because the esophageal temperature during the first $\mathrm{CB}$ application to the inferior PVs decreased below $15^{\circ} \mathrm{C}$, the application was ceased and superior PV isolation was achieved by the next application that was added after the esophageal temperature recovered to above $30^{\circ} \mathrm{C}$. Case presentation of a successful CST phenomenon: Case 1 involves a patient with paroxysmal AF who underwent de novo ablation with a CB (Figure 4). The LSPV was not isolated after the first $\mathrm{CB}$ application with an NT of $-52{ }^{\circ} \mathrm{C}$ and complete LSPV occlusion (Figure 4A). We did not add a further CB application to the LSPV but instead attempted LIPV ablation. At 21 seconds after initiating the ablation, both the LSPV and LIPV were simultaneously isolated (Figure 4B). Furthermore, frequent LIPV ectopic beats, which conducted to the LSPV without conducting to the LA, were observed. These electrophysiological findings could not be explained without the presence of an intervening connection between the LSPV and LIPV.

Case 2 involves a patient with persistent AF who underwent PV isolation with the $\mathrm{CB}$ (Figure 5). We confirmed the short AF cycle length in the LSPV and performed $\mathrm{CB}$ ablation to the PV (Figure 5A). Although the NT reached $-53^{\circ} \mathrm{C}$ during a $\mathrm{CB}$ application to the LSPV 

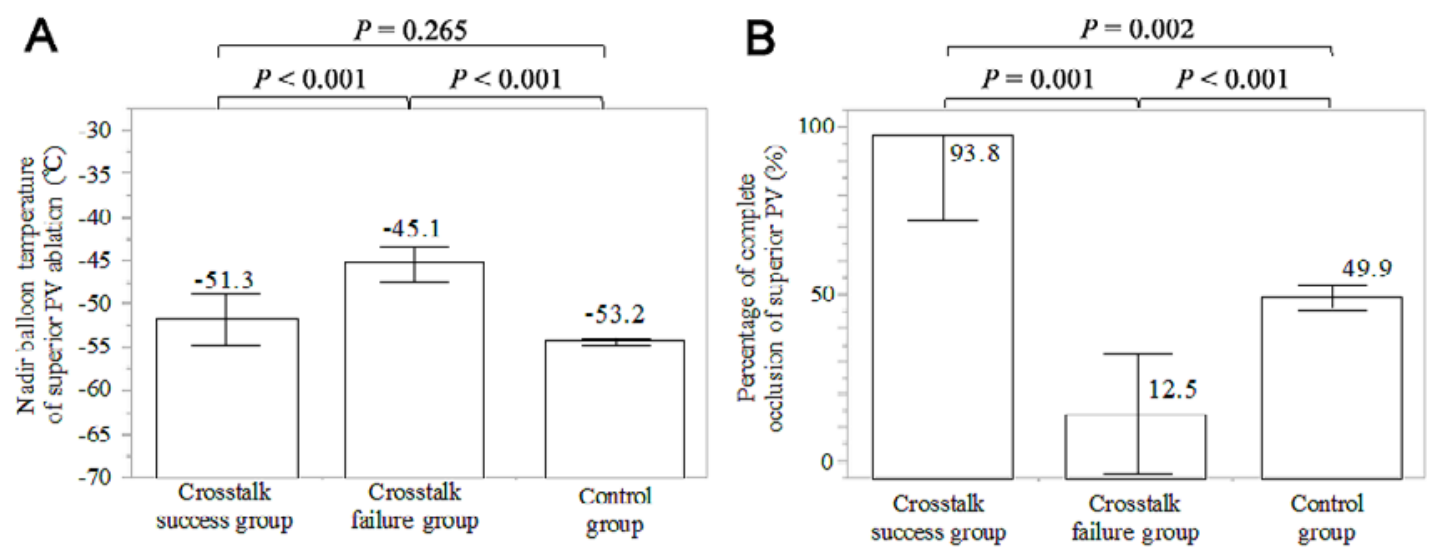

Figure 2. Comparison of factors during ablation among the three groups. A: The average NT was significantly lower in the CST success group than in the CST failure group. B: The percentage of complete occlusion of the superior PV with CB was significantly higher in the CST success group than in the other groups*. 95\% confidence intervals are shown in $\mathbf{A}$, whereas $98.3 \%$ confidence intervals are shown in $\mathbf{B}$. $* P<0.0167$ was considered significant, with adjustment by Bonferroni correction. CB indicates cryoballoon; CST, crosstalk; NT, nadir balloon temperature; and PV, pulmonary vein.

\section{- Crosstalk success group \\ N Crosstalk failure group}

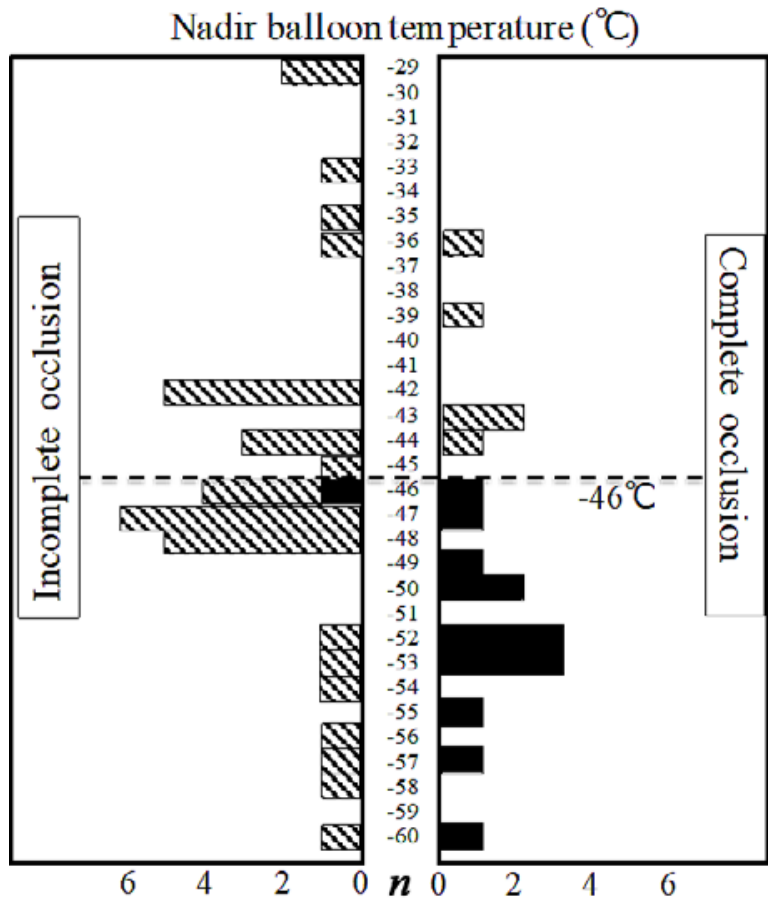

Figure 3. Distribution of the NT and the occlusion status of the superior PV among patients in the CST ablation success and the CST ablation failure groups. The NT in the CST ablation success group was mainly observed in the temperature range of $\leq-46^{\circ} \mathrm{C}$ when complete occlusion was achieved. In contrast, the NT in the CST ablation failure group was widely scattered throughout all temperatures, except in temperatures in which the CST ablation success group was mainly observed. CST indicates crosstalk; NT, nadir balloon temperature; and $\mathrm{PV}$, pulmonary vein.

and complete CB occlusion was obtained, the LSPV potential did not disappear. The AF cycle length in the
LSPV increased from $124 \mathrm{~ms}$ to $235 \mathrm{~ms}$ immediately after the $\mathrm{CB}$ application, but after several minutes, it shortened to $150 \mathrm{~ms}$. Although the LSPV was not isolated, complete occlusion of the LSPV was obtained; hence, we expected CST ablation and advanced the CB to the ipsilateral LIPV. As a result, the LSPV was isolated at 58 seconds after the freezing started. The CST was effective even though the AF cycle length in the LSPV was as short as $150 \mathrm{~ms}$ before ipsilateral LIPV CB ablation and LSPV potentials appeared to be largely unaffected to LSPV CB ablation.

\section{Discussion}

To the best of our knowledge, this is the first study to assess the CST phenomenon by performing precise electrophysiologic analysis. The major finding of this study was that in cases wherein $\mathrm{CB}$ ablation of the superior PVs was unable to isolate the PVs, the CST phenomenon was highly successful if $\mathrm{CB}$ ablation of the superior PVs met the following two criteria: NT $\leq-46^{\circ} \mathrm{C}$ and complete occlusion of the superior PVs. Furthermore, the CST phenomenon was mostly ineffective in cases wherein these two criteria were not met.

Generally, in CB ablation for LSPV, an operator often stops CB freezing when the PV is not electrically isolated even after 120 seconds from the start of freezing. However, CB freezing should not be stopped, but rather should be continued for 180-240 seconds when the ablation meets the above mentioned two criteria, because LSPV isolation is always successfully achieved following ipsilateral LIPV CB ablation.

Factors for an effective CST ablation: During CB ablation for PV isolation, PV potentials sometimes remain even if the targeted PV is perfectly occluded and the NT is low enough to freeze the PV ostium. Where previous studies reported that complete occlusion of the superior PVs was one of the proposed factors for an effective CST ablation; however, it was not statistically analyzed, ${ }^{13,14)}$ following the recognition of the CST phenomenon by Chun, 
Table II. Characteristics of PVs in the CST Success Group

\begin{tabular}{|c|c|c|c|c|c|c|c|c|c|c|c|c|c|c|}
\hline \multirow[b]{2}{*}{ Case } & \multicolumn{8}{|c|}{ CB application to SPV } & \multicolumn{6}{|c|}{ CB application to IPV } \\
\hline & $\begin{array}{c}\text { Cross- } \\
\text { talk } \\
\text { PV }\end{array}$ & $\begin{array}{l}\text { Com- } \\
\text { plete } \\
\text { Occlu- } \\
\text { sion }\end{array}$ & $\begin{array}{l}\mathrm{NT} \\
\left({ }^{\circ} \mathrm{C}\right)\end{array}$ & $\begin{array}{c}\text { Total } \\
\text { Freezing } \\
\text { Duration } \\
\text { (sec- } \\
\text { onds) }\end{array}$ & $\begin{array}{l}\text { Total } \\
\text { Freez- } \\
\text { ing } \\
\text { Time }\end{array}$ & $\begin{array}{c}\text { Tempo- } \\
\text { rary } \\
\text { PVI }\end{array}$ & $\begin{array}{l}\text { Duration } \\
\text { to } \\
\text { Tempo- } \\
\text { rary PVI } \\
\text { (sec- } \\
\text { onds) }\end{array}$ & $\begin{array}{l}\text { Time to } \\
\text { Superior } \\
\text { PV } \\
\text { Isolation } \\
\text { (sec- } \\
\text { onds) }\end{array}$ & $\begin{array}{l}\text { Time to } \\
\text { Inferior } \\
\text { PV } \\
\text { Isolation } \\
\text { (sec- } \\
\text { onds) }\end{array}$ & $\begin{array}{c}\mathrm{CB} \\
\text { Tem- } \\
\text { perature } \\
\text { at SPV } \\
\text { Isolation } \\
\left({ }^{\circ} \mathrm{C}\right)\end{array}$ & $\begin{array}{l}\text { Com- } \\
\text { plete } \\
\text { Occlu- } \\
\text { sion }\end{array}$ & $\begin{array}{c}\mathrm{NT} \\
\left({ }^{\circ} \mathrm{C}\right)\end{array}$ & $\begin{array}{c}\text { Total } \\
\text { Freezing } \\
\text { Duration } \\
\text { (seconds) }\end{array}$ & $\begin{array}{c}\text { Total } \\
\text { Freezing } \\
\text { Time }\end{array}$ \\
\hline 1 & LS & + & -55 & 155 & 1 & + & 65 & NR & NR & NA & + & -47 & 180 & 1 \\
\hline 2 & RS & + & -46 & 513 & 4 & - & & NR & NR & NA & + & NA & NA & 1 \\
\hline 3 & LS & + & -50 & 185 & 1 & - & & NR & 22 & NA & + & -48 & 168 & 1 \\
\hline 4 & LS & + & -52 & 542 & 3 & + & 145 & 233 & NR & -40 & - & -41 & 240 & 1 \\
\hline 5 & LS & + & -50 & 310 & 2 & - & & 21 & NR & -23 & - & -42 & 180 & 1 \\
\hline 6 & LS & + & -53 & 180 & 1 & + & 94 & 65 & NR & -36 & - & -42 & 180 & 1 \\
\hline 7 & LS & + & -53 & 180 & 1 & - & & 60 & 25 & -41 & + & -46 & 240 & 1 \\
\hline 8 & LS & + & -53 & 182 & 1 & - & & 24 & NR & -19 & - & -41 & 180 & 1 \\
\hline 9 & LS & + & -60 & 240 & 1 & + & 86 & 35 & 15 & -39 & - & -45 & 149 & 2 \\
\hline 10 & LS & + & -52 & 420 & 2 & - & & 46 & 46 & -36 & + & -45 & 121 & 1 \\
\hline 11 & LS & + & -47 & 180 & 1 & + & 97 & 31 & 59 & -35 & + & -42 & 180 & 1 \\
\hline 12 & LS & + & -52 & 180 & 1 & - & & 49 & NR & -35 & - & -43 & 153 & 1 \\
\hline 13 & LS & - & -46 & 180 & 1 & - & & NR & 26 & NA & + & -47 & 180 & 1 \\
\hline 14 & LS & + & -57 & 203 & 1 & + & 130 & 27 & 26 & -30 & + & -46 & 180 & 1 \\
\hline 15 & LS & + & -49 & 180 & 1 & - & & 40 & 39 & -40 & + & -53 & 180 & 1 \\
\hline 16 & LS & + & -48 & 180 & 2 & - & & 59 & 85 & -33 & + & -41 & 240 & 1 \\
\hline
\end{tabular}

CB indicates cryoballoon; LS, left superior; NA, not available; NR, not recorded during cryoballoon application; NT, nadir balloon temperature; $\mathrm{PV}$, pulmonary vein; PVI, pulmonary vein isolation; and RS, right superior.

et $a l .,{ }^{13)}$ the CST technique was introduced into the ablation setting. In this study, by comparing the ablation data among the CST success, failure, and control groups, we were able to obtain the sensitivity and specificity of factors for a gaining CST phenomenon and proposed two highly predictable factors for CST phenomenon-namely, $\mathrm{NT} \leq-46^{\circ} \mathrm{C}$ and complete PV occlusion during CB ablation of the superior PVs.

Clinical utility of the CST phenomenon: There can be an alternative to adding more $\mathrm{CB}$ applications to the present PV or shifting the $\mathrm{CB}$ to the ipsilateral PV in expectation of a CST phenomenon in cases with electrical connections remaining between the LA and PV despite rigorous $\mathrm{CB}$ applications to the superior PV. The success rate of PV isolation by the CST phenomenon was reported to be $41.9 \%(13 / 31$ cases $)$ by Miyazaki, et al. ${ }^{14)}$ and was $26.8 \%$ (15/56 patients) in our study, which is relatively low. Therefore, once the predictors postulated in this study could be introduced into the $\mathrm{CB}$ ablation setting, superior PV isolation would be expected to be more efficiently achieved, which would enable a reduction in the ablation application time and freezing damage to the myocardium and collateral tissues, including the esophagus and phrenic nerve. Despite the occurrence rate of CST phenomenon among all CB ablation cases being only $2.9 \%$ (16/541 patients) in our study and being reported to be $2.9 \%(15 / 511$ patients) and $4.7 \%$ (32/676 patients) in previous other studies, respectively, ${ }^{2,14}$ to the best of our knowledge, there has been no systematic study on how often the CST phenomenon can be observed in actual CB ablation.

The mechanisms of the CST phenomenon: The two distinct time differences required for the successful isolation of the superior and ipsilateral inferior PVs in this study would lead to the possibility of two mechanisms for the
CST phenomenon. As shown in Table II, the isolated cases were divided into two groups. In one group ("simultaneous" group), time to isolation of superior and inferior PV by the CB application to inferior PV were same (case $10)$, whereas in another group ("non-simultaneous" group), the superior PV was isolated with a nonsimultaneous but rather different timing from that of the ipsilateral inferior PV, as in cases 7, 9, 11, 14, 15, and 16 . Based on these findings, two mechanisms could be proposed, as delineated in Figure 6. An epicardial connection intervening between the ipsilateral PVs (epicardial conduction) may exist in the "simultaneous" group. On the other hand, the sleeve myocardium in the carina region was sometimes so thick that LSPV CB ablation alone cannot ablate the myocardium in the carina region over the entire layer, which resulted in unsuccessful LSPV isolation (carina conduction). In "non-simultaneous" group, it is likely that the outer part of the myocardium in this thick carina region would have to be ablated from the LIPV side to achieve full-layered ablation for LSPV isolation. There are no specific differences of patient characteristics and long-term success rate between the two groups.

The prevalence of an epicardial conduction was reported by previous studies to be $13.5 \%-20 \%$. $^{16-18)}$ While Takahashi, et al. reported that out of 49 cases in which the LSPV was difficult to isolate by radiofrequency catheter ablation, seven cases had an earliest activation site in the LIPV during intra-LSPV pacing, which suggested an epicardial conduction between the ipsilateral $\mathrm{PVs},{ }^{20)}$ to date, various epicardial conduction paths between the PVs and other atrial structures have been reported: LSPVposterior LA, LSPV-vein of Marshall (VOM) area, LSPVLA roof, left common PV (LCPV)-VOM area, LCPVposterior LA, RSPV-posterior LA, and RSPV-LA roof. ${ }^{16-21)}$ 
A

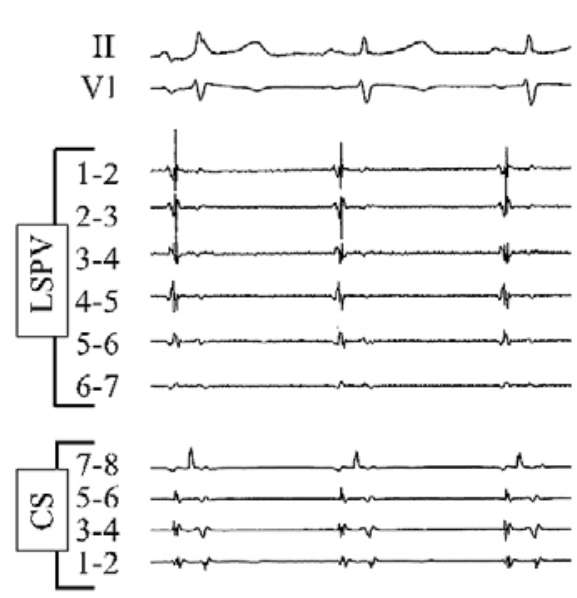

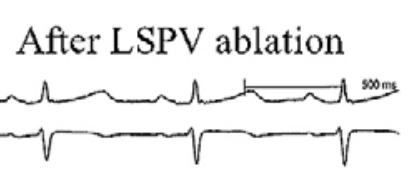

LSPV CB ablation

Complete Occlusion

240 seconds, NT: $-52^{\circ} \mathrm{C}$

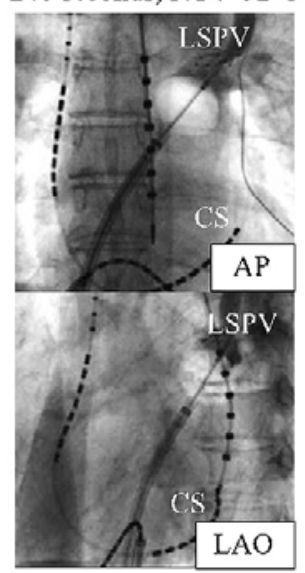

\section{B}

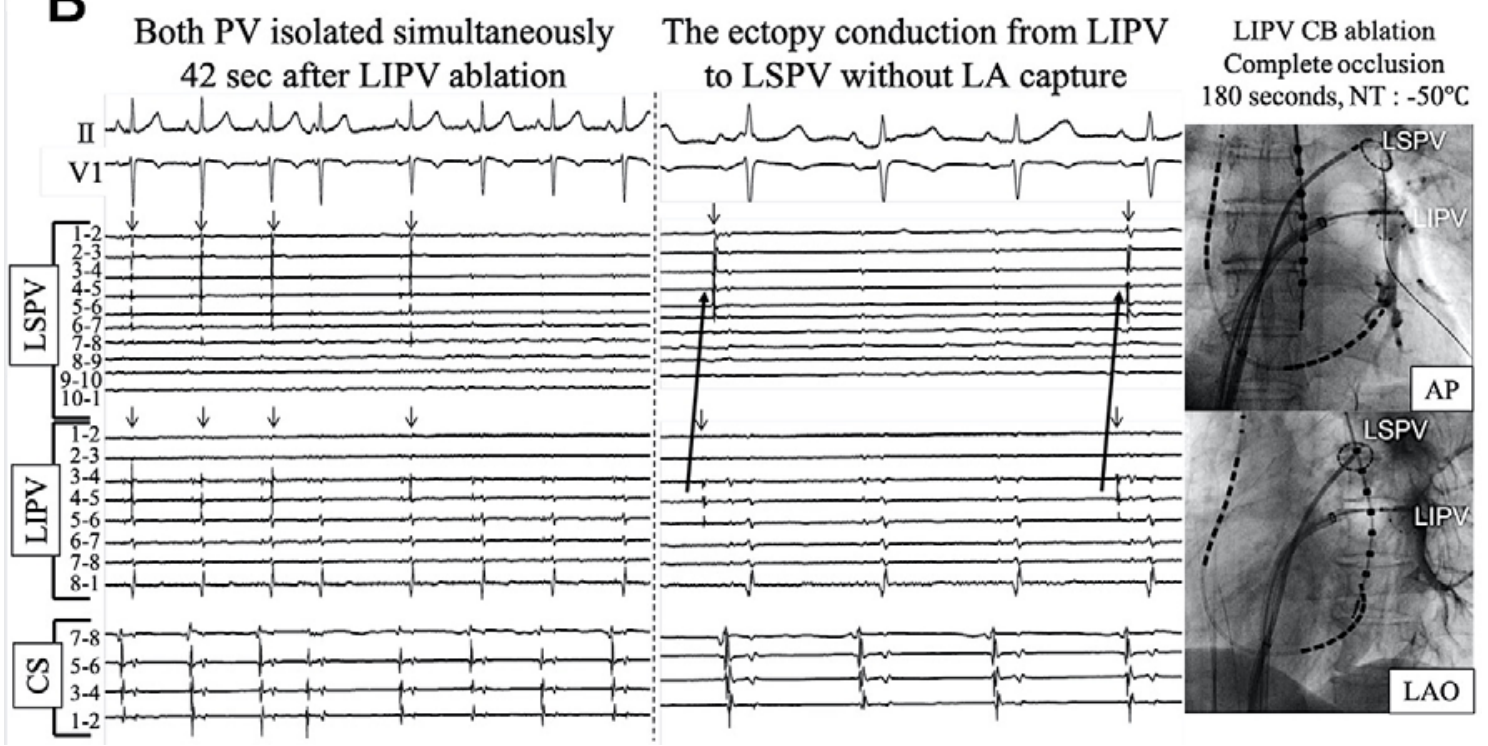

Figure 4. Successful CST ablation case. A: LSPV isolation was not achieved by CB ablation with an NT of $52^{\circ} \mathrm{C}$, complete LSPV occlusion, and freezing duration of 240 seconds. B: Successful CST ablation during CB ablation of the LIPV was observed. Both LSPV and LIPV were isolated simultaneously and LIPV ectopic beats conducted to the LSPV without conducting to the LA. AP indicates anterior-posterior; CB, cryoballoon; CS, coronary sinus; CST, crosstalk; LA, left atrium; LAO, left anterior oblique; LSPV, left superior pulmonary vein; LIPV, left inferior pulmonary vein; and NT, nadir balloon temperature.

The durability of acute PV isolation and the longterm AF survival rate have been reported to be lower in cases with epicardial conduction than in cases without such conduction. ${ }^{16,17)}$ In our study, no acute electrical reconnections of the PVs were documented in the CST success group.

Anatomical reports have described that the thickest myocardial sleeves around the PVs are found in the carina region. ${ }^{22,23)}$ Cabrera, et al. reported these connections from an anatomical aspect. ${ }^{23,24)}$ In histologic sections of 15 hearts, $27 \%, 53 \%$, and $20 \%$ of myocardial strands between the superior and inferior PVs were epicardial, subendocardial, and from both aspects of the PV wall, re- spectively. The strands on the epicardial PV wall are likely to correspond to electrical epicardial intervening connections. That anatomical aspect of the PVs may account for the two conduction patterns observed during CB applications and eventual CST phenomenon.

Study limitations: In our study population, the prevalence of the CST phenomenon was observed in 16 (3\%) patients, which is a small sample size and may not have been adequate to derive any accurate factors for predicting a CST phenomenon. Additionally, whether the two factors derived could be useful predictors in the $\mathrm{CB}$ ablation setting was not evaluated owing to the retrospective nonrandomized design of this study. Hence, these two factors 

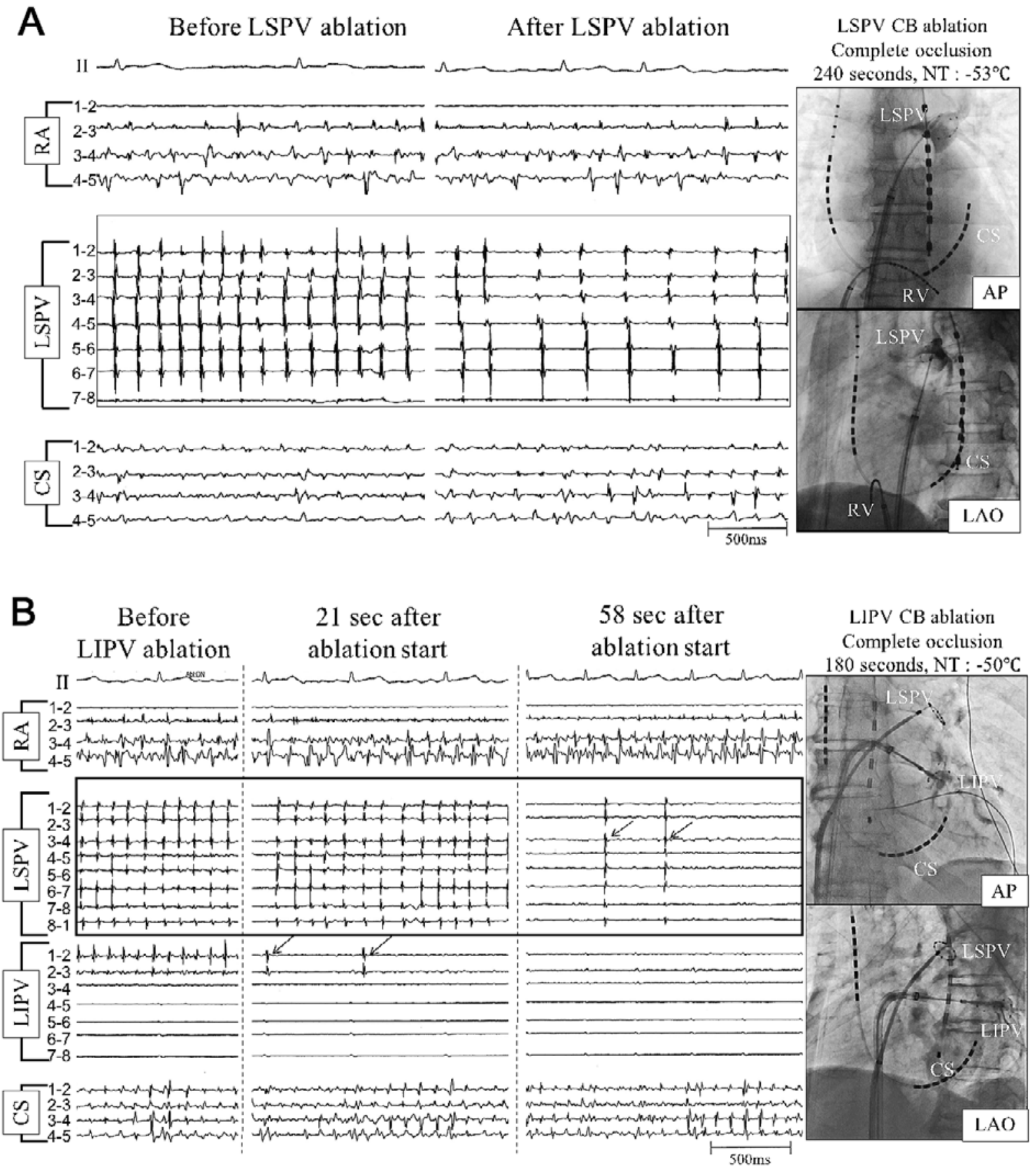

Figure 5. Successful CST ablation case. A: LSPV isolation was not achieved by CB ablation with an NT of $-53^{\circ} \mathrm{C}$, complete LSPV occlusion, and freezing duration of 240 seconds. B: Successful CST ablation during CB ablation of the LIPV was observed even if the AF cycle length was as short as $150 \mathrm{~ms}$ before the ablation. AF indicates atrial fibrillation; AP, anterior-posterior; $\mathrm{CB}$, cryoballoon; CS, coronary sinus; CST, crosstalk; LAO, left anterior oblique; LSPV, left superior pulmonary vein; LIPV, left inferior pulmonary vein; and NT, nadir balloon temperature.

should be evaluated in a prospective, randomized, multicenter study in the future.

\section{Conclusion}

A CST phenomenon was highly predicted if the NT was $\leq-46^{\circ} \mathrm{C}$ and a complete $\mathrm{PV}$ occlusion during $\mathrm{CB}$ ab- lation of the superior PVs were achieved. Knowing these predictors would help operators reduce unnecessary freezing during superior PV isolation using a CB.

\section{Acknowledgment}

We thank Mr. John Martin for his linguistic support. 

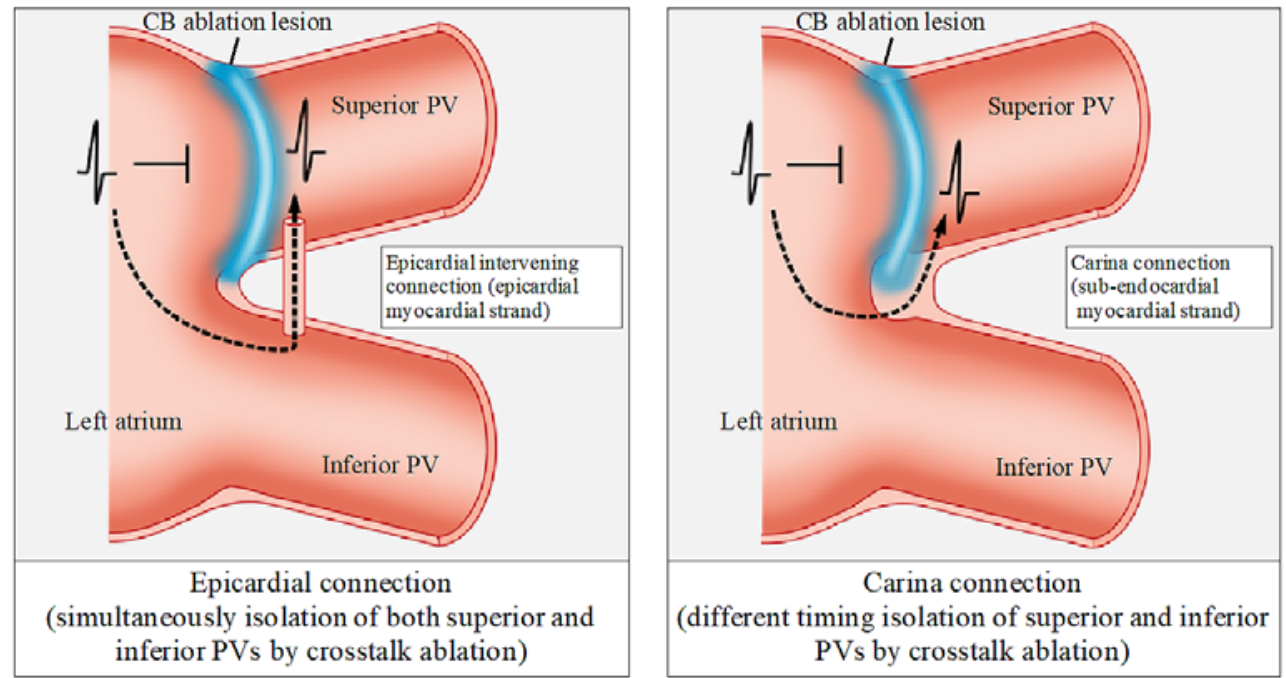

Figure 6. The speculated mechanisms of CST phenomenon. There are two possible mechanisms for the CST phenomenon. CB indicates cryoballoon; CST, crosstalk; and PV, pulmonary vein.

\section{Disclosure}

\section{Conflicts of interest: None.}

\section{References}

1. Kuck KH, Brugada J, Fürnkranz A, et al. Cryoballoon or radiofrequency ablation for paroxysmal atrial fibrillation. $\mathrm{N}$ Engl J Med 2016; 374: 2235-45.

2. Yang JD, Sun Q, Guo XG, et al. 'Crosstalk' technique: a comparison between two generations of cryoballoon catheter. Pacing Clin Electrophysiol 2018; 41: 635-42.

3. Mörtsell D, Arbelo E, Dagres N, et al. Cryoballoon vs. radiofrequency ablation for atrial fibrillation: a study of outcome and safety based on the ESC-EHRA atrial fibrillation ablation longterm registry and the Swedish catheter ablation registry. Europace 2019; 21: 581-9.

4. Murray MI, Arnold A, Younis M, Varghese S, Zeiher AM. Cryoballoon versus radiofrequency ablation for paroxysmal atrial fibrillation: a meta-analysis of randomized controlled trials. Clin Res Cardiol 2018; 107: 658-69.

5. Aryana A, Singh SM, Kowalski M, et al. Acute and long-term outcomes of catheter ablation of atrial fibrillation using the second-generation cryoballoon versus open-irrigated radiofrequency: a multicenter experience. J Cardiovasc Electrophysiol 2015; 26: 832-9.

6. Neumann T, Vogt J, Schumacher B, et al. Circumferential pulmonary vein isolation with the cryoballoon technique results from a prospective 3-center study. J Am Coll Cardiol 2008; 52: 273-8.

7. Knight BP, Novak PG, Sangrigoli R, et al. Long-term outcomes after ablation for paroxysmal atrial fibrillation using the secondgeneration cryoballoon: final results from STOP AF postapproval study. JACC Clin Electrophysiol 2019; 5: 306-14.

8. Tokutake K, Tokuda M, Ogawa T, Matsuo S, Yoshimura M, Yamane T. Pulmonary vein stenosis after second-generation cryoballoon ablation for atrial fibrillation. HeartRhythm Case Rep 2017; 3: 36-9

9. Watanabe K, Nitta J, Sato A, Goya M, Isobe M, Hirao K. Hemoptysis after five months of cryoballoon ablation: what is the relationship? HeartRhythm Case Rep 2017; 3: 357-9.

10. John RM, Kapur S, Ellenbogen KA, Koneru JN. Atrioe- sophageal fistula formation with cryoballoon ablation is most commonly related to the left inferior pulmonary vein. Heart Rhythm 2017; 14: 184-9.

11. Verma N, Gillespie CT, Argento AC, et al. Bronchial effects of cryoballoon ablation for atrial fibrillation. Heart Rhythm 2017; 14: 12-6.

12. Schweigert M, Almeida AB. Life-threatening pulmonary haemorrhage during cryoballoon ablation for atrial fibrillation. Eur J Cardio Thorac Surg 2018; 54: 789-91.

13. Chun KR, Schmidt B, Metzner A, et al. The "single big cryoballoon" technique for acute pulmonary vein isolation in patients with paroxysmal atrial fibrillation: a prospective observational single centre study. Eur Heart J 2009; 30: 699-709.

14. Miyazaki S, Kajiyama T, Watanabe T, et al. Impact of electrical connections between ipsilateral pulmonary veins on the secondgeneration cryoballoon ablation procedure. J Cardiovasc Electrophysiol 2019; 30: 27-31.

15. Okishige K, Aoyagi H, Kawaguchi N, et al. Novel method for earlier detection of phrenic nerve injury during cryoballoon applications for electrical isolation of pulmonary veins in patients with atrial fibrillation. Heart Rhythm 2016; 13: 1810-6.

16. Pérez-Castellano N, Villacastín J, Salinas J, et al. Epicardial connections between the pulmonary veins and left atrium: relevance for atrial fibrillation ablation. J Cardiovasc Electrophysiol 2011; 22: 149-59.

17. Barrio-Lopez MT, Sanchez-Quintana D, Garcia-Martinez J, et al. Epicardial connections involving pulmonary veins: the prevalence, predictors, and implications for ablation outcome. Circ Arrhythm Electrophysiol 2020; 13: e007544

18. Yoshida K, Baba M, Shinoda Y, et al. Epicardial connection between the right-sided pulmonary venous carina and the right atrium in patients with atrial fibrillation: a possible mechanism for preclusion of pulmonary vein isolation without carina ablation. Heart Rhythm 2019; 16: 671-8.

19. Tritto M, De Ponti R, Zardini M, Spadacini G, Oliveira M, Salerno-Uriarte JA. Electrical connections between pulmonary veins in humans: evidence after radiofrequency ablation of the venoatrial junction. Circulation 2001; 104: E30-1.

20. Takahashi A, Iesaka Y, Takahashi Y, et al. Electrical connections between pulmonary veins: implication for ostial ablation of pulmonary veins in patients with paroxysmal atrial fibrillation. Circulation 2002; 105: 2998-3003.

21. Matsuo S, Yamane T, Tokuda M, et al. The dormant epicardial 
reconnection of pulmonary vein: an unusual cause of recurrent atrial fibrillation after pulmonary vein isolation. Pacing Clin Electrophysiol 2008; 31: 920-4.

22. Mulder MJ, Kemme MJB, Götte MJW, et al. Differences between gap-related persistent conduction and carina-related persistent conduction during radiofrequency pulmonary vein isolation. J Cardiovasc Electrophysiol 2020; 31: 1616-27.

23. Ho SY, Cabrera JA, Tran VH, Farré J, Anderson RH, Sánchez-
Quintana D. Architecture of the pulmonary veins: relevance to radiofrequency ablation. Heart 2001; 86: 265-70.

24. Cabrera JA, Ho SY, Climent V, Fuertes B, Murillo M, SánchezQuintana D. Morphological evidence of muscular connections between contiguous pulmonary venous orifices: relevance of the interpulmonary isthmus for catheter ablation in atrial fibrillation. Heart Rhythm 2009; 6: 1192-8. 